\title{
Heparinization of gas plasma-modified polystyrene surfaces and the interactions of these surfaces with proteins studied with surface plasmon resonance
}

\author{
C.J. van Delden, J.P. Lens, R.P.H. Kooyman*, G.H.M. Engbers \\ and J. Feijen \\ University of Twente, Department of Chemical Engineering, Biomaterials Section, P.O. Box 217, 7500 AE, Enschede \\ The Netherlands and the Institute for Blomedical Technology (BMTI), Enschede, The Netherlands; 'Department of \\ Applied Physics, Applied Optics Group, University of Twente, PO Box 217, 7500AE Enschede, The Netherlands
}

\begin{abstract}
Polystyrene surfaces obtained by spin-coating a solution of polystyrene in toluene on a gold layer were functionalized with carboxylic acid groups by preadsorption of the sodium salt of undecylenic acid, followed by an argon plasma treatment. A conjugate of albumin and heparin (alb-hep) was covalently immobilized onto the functionalized surface via preactivation of carboxylic acid groups with a water-soluble carbodiimide. The immobilization of alb-hep conjugate and the subsequent interactions of the heparinized surface with antithrombin III (ATIII, a heparin cofactor) and thrombin were monitored with surface plasmon resonance (SPR). The surface concentration of conjugate as determined with SPR deviated quantitatively from the results obtained with radiolabelled conjugate. The difference in surface concentrations of conjugate obtained with the two methods probably originates from the uncertainty of the refractive index of the alb-hep conjugate in the SPR technique. ATIII could be bound to the surface modified with alb-hep conjugate but not to a polystyrene surface modified with albumin. Rabbit anti-human ATIII did bind to the alb-hep surface previously exposed to ATIII, confirming the presence of surface bound ATIII. The alb-hep immobilized surface was able to bind much more thrombin than ATIII, which is probably due to the less specific heparin-thrombin interaction as compared to the heparin-ATIII interaction. This study shows that SPR is a technique that can be used to study, in real time, both the modification of polymer surfaces and the subsequent interactions of the modified surfaces with proteins. (C) 1997 Elsevier Science Limited. All rights reserved
\end{abstract}

Keywords: Surface plasmon resonance, heparinization, protein-surface interactions

Received 30 October 1995; accepted 19 November 1996

The interactions between proteins and the surface of biomaterials has been the subject of many studies. In these studies, radiolabelling of proteins has frequently been used because of the high sensitivity of the method. The introduction of a label, however, may affect the conformation and biological activity of the protein. After the labelling procedure the influence of the label on the protein properties has to be checked. Furthermore, protein-surface interactions cannot be determined continuously when proteins are labelled with, for instance, a beta-emitter as carbon-14. In the present study, a functionalized polystyrene surface has been modified with albumin-heparin (alb-hep) conjugate. The immobilization of conjugate and the subsequent interactions of this heparinized surface

Correspondence to Dr J. Feijen. with antithrombin III (ATII) and thrombin have been investigated by means of surface plasmon resonance (SPR), a non-destructive optical method. The results obtained with SPR have been compared with the results obtained using radiolabelled proteins. Heparinization of polymer surfaces is a well known technique to enhance the blood compatibility of polymer surfaces. Heparin is an anticoagulant which displays its activity via cofactors, predominantly ATIII. Reflectometry, ellipsometry, total internal reflection fluorescence and also SPR are optical methods that can be applied in a bio- or immuno-sensor. With these techniques, surface-protein interactions can be followed in real time, without the use of labels. In contrast to some other methods, both the incident and the reflected light in the SPR apparatus do not pass the medium in contact with the sensor surface. The SPR apparatus 
and equipment are relatively small and inexpensive. The use of SPR as a biosensor was first described in $1983^{1}$. This application has gained a lot of interest, especially after Pharmacia launched a new Biospecific Interaction Analyser (BIAcore ${ }^{\circledR}$ ) based on SPR in $1990^{2}$. SPR is a phenomenon that arises as a sharp minimum in the intensity of the reflected light when the incident beam is introduced at a certain angle on a metal layer ${ }^{3}$. Although the incident light is totally internally reflected, an electromagnetic field component of the light (the evanescent wave) penetrates the metal layer. The evanescent wave can couple to a surface plasmon, an oscillation of electrons at the metal interface. This coupling is achieved at a specific angle of incidence. At this angle the reflectance goes through a minimum due to the SPR. The angle of the reflected light at which the SPR is observed is highly sensitive to changes in the refractive index of a thin layer $( \pm 50-100 \mathrm{~nm})$ in the direct vicinity of the metal surface. When proteins adsorb to the metal surface, an increase of the concentration of proteins near the surface occurs, resulting in an increase of the mean refractive index in the probe volume near the surface. The increase in refractive index leads to a shift of the SPR angle to higher values (Figure 1).

The magnitude of the angular shift depends on the change of the mean refractive index due to the increase of the protein concentration in the probe volume upon protein adsorption to the surface. Since most proteins have a similar refractive index, the SPR shift can be converted to an average thickness of an additional protein layer on the metal surface. To this end, our procedure $^{7}$ was to solve Fresnel's equations for the stratified layer system prism/gold/protein layer/water, with the measured SPR angle minimum as input variable and the protein layer thickness as unknown. Stenberg et al. ${ }^{4}$ used the BIAcore and several radiolabelled proteins to show that the SPR shift due to protein adsorption was proportional to the surface concentration of the adsorbed proteins as determined with radioactivity measurements. SPR is also very suitable for monitoring ligand-receptor interactions, especially when ligands can be immobilized covalently onto the sensor surface. In the BIAcore of Pharmacia, a 100-nm-thick carboxylated dextran layer, covalently immobilized on a gold layer, is used. The carboxylic acid groups of the dextran layer can be activated and used for the immobilization of ligands. In the past few years, SPR has proven to be a powerful biosensing technique which is sensitive (detection limit $\pm 1 \%$ of a monolayer of adsorbed protein), does not require a label and allows the monitoring in real time ${ }^{8,17}$. In this study, an SPR apparatus was used which was developed at the University of Twente. Detailed descriptions of the instrument have been published previously (see Figure 1 and the references cited therein).

The aim of the present investigation is to evaluate whether SPR in combination with surface plasma treatment can be used to study both the heparinization of a polymer surface as well as the subsequent interactions between this heparinized surface and ATIII and thrombin in real time.

\section{MATERIALS AND METHODS}

\section{Materials}

Unfractionated alb-hep conjugate containing $16.5 \%$ heparin $(w / w)$ was purchased from HBG (Enschede, The Netherlands). Purified human ATIII was a gift from the Central Laboratory of the Netherlands Red Cross Transfusion Service (CLB; Amsterdam, the Netherlands). Phosphate-buffered saline (PBS; $\mathrm{pH}$ 7.4) was obtained from NPBI (Emmercompascuum, The Netherlands). Sodium dodecyl sulphate (SDS), rabbit anti-human ATIII and rectangular glass slides $\left(20 \times 26 \times 0.4 \mathrm{~mm}^{3}\right)$ were purchased from Merck (Darmstadt, Germany). N-Hydroxysuccinimide (NHS) was purchased from Fluka (Buchs, Switzerland). Bovine thrombin ( $300 \mathrm{Umg}^{-1}$ ), bovine albumin and the sodium salt of undecylenic acid [C11(:)] were obtained from Sigma (St Louis, MO, USA). $\mathrm{N}$-(3Dimethylaminopropyl)- $N^{\prime}$-ethylcarbodiimide (EDC) was purchased from Merck-Schuchardt (Hohenbrunn, Germany). Argon (Ar; purity $>99.999 \%$ ) was obtained from Hoekloos (Amsterdam, The Netherlands). Round glass slides (diameter $25 \mathrm{~mm}$, thickness $1 \mathrm{~mm}$ ) were purchased from Balzers (Maarssen, The Netherlands).

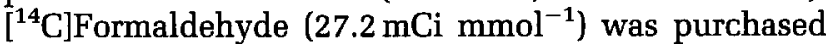
from Amersham (Amersham, UK). Polystyrene type $680 \mathrm{E}$ was a gift from Dow Chemicals (Terneuzen, The Netherlands).

\section{Introduction of carboxylic acid groups onto polystyrene-coated surfaces by an argon plasma treatment}

For practical reasons, in this experiment and in the experiment for the coupling of labelled alb-hep conjugate onto the modified surfaces, rectangular glass slides were used which were dip coated in a
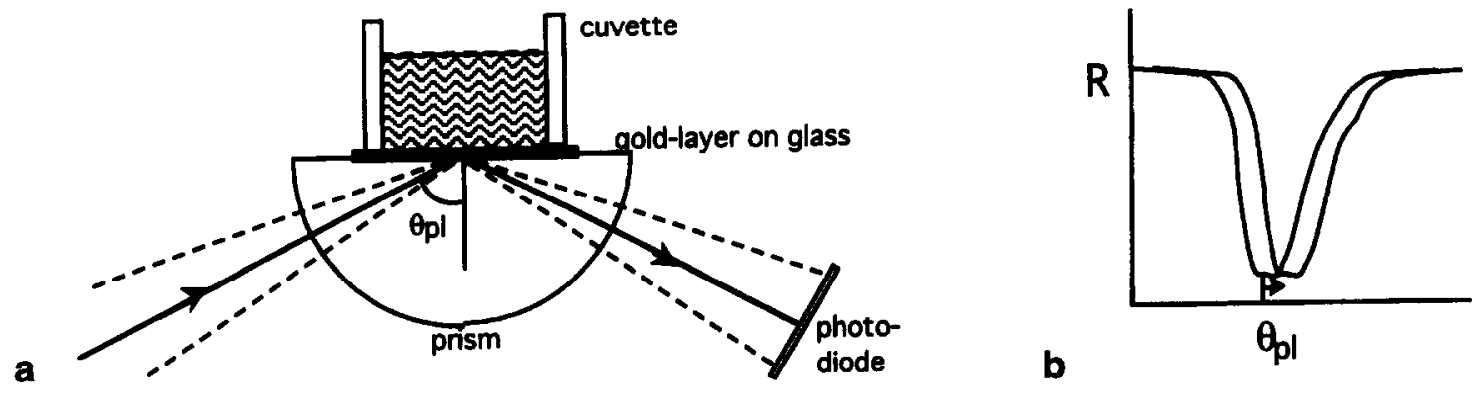

Figure $1 \mathrm{a}$, The sensor unit of the SPR apparatus. $\mathbf{b}$, The reflection minimum observed at plasmon angle $\theta_{\mathrm{pl}}$ shifts to a higher value, when for instance, proteins are adsorbed on the gold layer. The actual apparatus, which combines a cylindrical prism and a vibrating mirror, has been described previously ${ }^{5,6}$. 
polystyrene (PS) solution. For the SPR experiment round, gold-coated glass slides were used, which were spun cast with PS.

Rectangular glass slides were cleaned in a chromic acid solution for at least $24 \mathrm{~h}$, after which they were washed extensively with ultrapure water and stored in absolute ethanol. Before use, the glass slides were dried in air. The slides were dipped into a $0.2 \%(\mathrm{w} / \mathrm{v})$ PS solution in toluene and dried at room temperature for at least $24 \mathrm{~h}$. C11(:) was immobilized onto polystyrene-coated glass slides (PS surfaces) by means of an argon plasma treatment. First C11(:) was adsorbed onto PS surfaces by immersing PS-containing glass slides in an aqueous solution of C11(:) $(0.3 \mathrm{M})$ containing $1 \%(\mathrm{v} / \mathrm{v})$ hexanol. After $5 \mathrm{~min}$, the solution was removed and the samples were dried in vacuo. PS and C11(:) preadsorbed samples [PS/C11(:)] were subsequently treated with an argon plasma for $5 \mathrm{~s}$ at $45 \mathrm{~W}$ and $0.08 \mathrm{mbar}$, with no gas flow through the reactor during plasma treatment. The reactor configuration is similar to that described by Terlingen et al. ${ }^{9}$. The plasma-treated samples PS/C11(:)-Ar and PS-Ar were washed with water and dried in vacuo: PS/C11(:)-Ar$\mathrm{H}_{2} \mathrm{O}$ and $\mathrm{PS}-\mathrm{Ar}-\mathrm{H}_{2} \mathrm{O}$.

The elemental composition of the surfaces was determined with X-ray photoelectron spectroscopy (XPS) measurements (Kratos XSAM-800, Manchester, UK). In order to detect carboxylic acid groups on the washed, plasma-treated samples they were subjected to a gas phase reaction with trifluoroethanol (TFE). This method was first described by Chilkoti et al. ${ }^{10}$ and has been modified by Terlingen et al. ${ }^{11}$. In short, plasma treated samples were placed vertically in a glass vial on glass beads. TFE $(0.45 \mathrm{ml})$, pyridine $(0.2 \mathrm{ml})$ and 1,3-di-tert-butylcarbodiimide $(0.15 \mathrm{ml})$ were injected into the vial. The reaction in the sealed vial was allowed to proceed for $24 \mathrm{~h}$ at room temperature, after which the XPS measurements were performed.

\section{Immobilization of $\left[{ }^{14} \mathrm{C}\right]$ alb-hep conjugate on (functionalized) PS surfaces - alb-hep surface concentration and stability}

Glass slides were dipped onto a $0.2 \%$ PS ( $/ / v)$ solution in toluene, after which C11(:) was adsorbed onto the PS surfaces as described above. Unmodified (PS) and PS/ C11(:) samples were treated with a static argon plasma for $5 \mathrm{~s}$. PS surfaces which were not treated with an argon plasma were used as a negative control surface in this experiment. After the plasma treatment, the PS samples were washed with water for at least $30 \mathrm{~min}$. Alb-hep conjugate was labelled with $\left[{ }^{14} \mathrm{C}\right]$ formaldehyde $^{12}$ to study the surface concentration and stability of alb-hep conjugate immobilized on the modified PS surfaces. $\left[{ }^{14} \mathrm{C}\right] \mathrm{Alb}-\mathrm{hep}$ conjugate was coupled onto PS-Ar- $\mathrm{H}_{2} \mathrm{O}$ and PS/C11(:)-Ar- $\mathrm{H}_{2} \mathrm{O}$ using EDC in the presence of NHS. The carboxylic acid groups at the modified PS surfaces were first activated in a $\mathrm{NaH}_{2} \mathrm{PO}_{4}$ buffer ( $5 \mathrm{mM}, \mathrm{pH} 4.6$ ) containing $1 \mathrm{mM}$ EDC and NHS. After $30 \mathrm{~min}$ the surfaces that contained activated carboxylic acid groups were placed in a borate buffer $(5 \mathrm{mM}, \mathrm{pH} 8.2)$ which contained $15 \mathrm{mg} \mathrm{ml}^{-1}\left[{ }^{14} \mathrm{C}\right]$ alb-hep conjugate. After immersing the surfaces in the $\left[{ }^{14} \mathrm{C}\right]$ alb-hep conjugate solution, aliquots of EDC and NHS were added, resulting in a final concentration of $0.15 \mathrm{mM}$ for both reagents. The coupling reaction was allowed to proceed for $\pm 1 \mathrm{~h}$, whereafter the surfaces were washed with PBS (three times), with a phosphate buffer (12 $\mathrm{mM})$ containing $1 \mathrm{M}$ $\mathrm{NaCl}$ with a pH of 3.5 (three times) and with a similar solution of $\mathrm{pH} 10.5$ (three times). Finally, the surfaces were washed twice with PBS. Half of the samples were directly transferred into a scintillation vial, whereas the other half was incubated in a $3 \%$ SDS solution for $18 \mathrm{~h}$ to desorb labelled conjugate that was not covalently immobilized. After the desorption procedure, the SDS-treated samples were dipped dry on a tissue and transferred to a scintillation vial. All samples were incubated in $2 \mathrm{ml}$ LumaSolve at $55^{\circ} \mathrm{C}$ for $1 \mathrm{~h}$ to dissolve the (remaining) labelled conjugate from the surface. The vials were cooled to room temperature and $18 \mathrm{ml}$ LumaGel and $200 \mu \mathrm{l} 6 \mathrm{~N} \mathrm{HCl}$ were added. Radioactivity of the samples was determined with a scintillation counter (Winspectral 1414, Wallac, Turku, Finland). The radioactivity was converted into a surface concentration of alb-hep conjugate by dividing the cpm values by the surface area used per experiment and the specific activity of the conjugate (cpm per $\mu \mathrm{g}$ ).

\section{Immobilization of alb-hep conjugate on function- alized PS surfaces and the interaction of ATIII and thrombin with surface immobilized alb-hep conjugate studied with SPR}

Round glass slides containing layers of $2 \mathrm{~nm}$ chromium and $47.8 \mathrm{~nm}$ gold were spin-coated using a PS solution $(0.10 \% \mathrm{w} / \mathrm{v}$ in toluene). The PS solution $(30 \mu \mathrm{l})$ was pipetted on the gold layer and spread out with a Pasteur pipette. The sample was stirred for $2 \mathrm{~min}$ at $2000 \mathrm{rpm}$ and then dried for $24 \mathrm{~h}$ at room temperature The samples were immersed in a C11(:)-containing solution, dried in vacuo and subsequently treated with an argon plasma as described above. The samples were washed with water, dried in vacuo and stored under nitrogen. The PS layer thickness on a gold surface spin-coated with a $0.10 \%$ polymer solution was determined with SPR. The plasmon angle of uncoated gold surfaces was compared with the plasmon angle of PS-coated samples. The observed plasmon angle difference was converted to a layer thickness of $15 \pm 1 \mathrm{~nm}$ assuming a refractive index for polystyrene of $1.59^{12}$.

The covalent immobilization of alb-hep conjugate was performed in the SPR apparatus, which enabled us to follow the immobilization in time. A PS/C11(:)$\mathrm{Ar}-\mathrm{H}_{2} \mathrm{O}$ sample was placed in the apparatus. A rubber gasket and a Teflon cuvette which contained two compartments were placed on the sample, after which $1 \mathrm{ml}$ PBS was added to both compartments to obtain a baseline. The PBS was partially replaced by an $\mathrm{NaH}_{2} \mathrm{PO}_{4}$ solution ( $5 \mathrm{mM}$, pH 4.6). Aliquots of EDC and NHS were added, resulting in a final concentration for both components of $1 \mathrm{mM}$. After $20 \mathrm{~min}$, the phosphate buffer was replaced by a borate buffer $(5 \mathrm{mM}, \mathrm{pH} 8.4)$, containing $15 \mathrm{mg} \mathrm{m}^{-1}$ alb-hep conjugate or bovine albumin. Since the alb-hep conjugate was prepared by the supplier via coupling of the heparin to the albumin in the presence of EDC, the albumin used in this study was pretreated with EDC under the same conditions as the albumin in the conjugate ${ }^{13}$. In contrast to the albhep conjugate, EDC-treated albumin was not chromato- 
graphically purified but was directly dialysed against water and then freeze dried. A small amount of the phosphate buffer was left in the cuvettes, which resulted in an EDC and NHS concentration of $\pm 0.1 \mathrm{mM}$ after replacing the phosphate buffer with the borate buffer. The immobilization was followed in time and stopped after about $40 \mathrm{~min}$ by replacing the borate solution with PBS. The surfaces were washed with PBS (three times), with a phosphate buffer (12 mM) containing $1 \mathrm{M} \mathrm{NaCl}(\mathrm{pH} 3.5$; three times) and with a phosphate buffer (12 mM, pH 10.5; three times). Finally, the last washing solution was replaced by PBS. After a constant SPR signal was obtained, ATIII $\left(100 \mu \mathrm{g} \mathrm{ml}^{-1}\right)$, dissolved in PBS, was added to both compartments. The ATII uptake by the alb-hep and albumin surface was followed for $15 \mathrm{~min}$, after which ATIII was replaced by PBS. Thereafter, thrombin was added to the PBS solution in both compartments, resulting in a final concentration of $12.5 \mathrm{U} \mathrm{ml}^{-1}$. It must be noticed that after the addition of conjugate or protein to the cuvette the solution in the cuvette was homogenized by filling and emptying several times the same pipette that was used for adding the protein solution.

In a second experiment, alb-hep conjugate and albumin were immobilized, each in one compartment, onto PS/C11(:)-Ar- $\mathrm{H}_{2} \mathrm{O}$ according to the same procedure as described above. After immobilization and washing of the surfaces, ATII $\left(100 \mu \mathrm{g} \mathrm{ml}^{-1}\right)$ was added to both compartments. The ATIII solution was replaced by PBS, after which the surfaces were washed with PBS to remove lonsely bound ATIII. Finally, polyclonal immunoglobulin against human ATIII was added to the PBS solution.

\section{RESULTS}

\section{Surface analysis}

The results of the XPS analyses of the plasma-treated and control surfaces are presented in Table 1. All measurements were performed in duplicate except for PS-Ar, of which only one sample was analysed. Compared to a bare PS surface, all modified surfaces had a higher oxygen percentage. PS surfaces exposed to $\mathrm{C} 11($ :) which were not plasma treated contained about $6 \%$ oxygen on the surface, whereas samples that were plasma treated contained the highest oxygen content. The percentage of oxygen detected on the argon-treated PS and PS/C11(:) samples was almost the same. The amount of fluorine after reaction with TFE is an indication of the amount of carboxylic acid groups. The fluorine content was somewhat higher on the PS/C11(:)-Ar- $\mathrm{H}_{2} \mathrm{O}-\mathrm{TFE}$ than on PS-Ar- $\mathrm{H}_{2} \mathrm{O}-\mathrm{TFE}$ samples. Washing of plasma-treated surfaces with water did not result in a change in the atom percentage of oxygen.

When it is assumed that all the carboxylic acid groups that are introduced by the plasma treatment are present at the surface, the carboxylic acid content of PS/C11(:)-Ar- $\mathrm{H}_{2} \mathrm{O}-\mathrm{TFE}$ corresponds with an average surface area per carboxylic acid group of $\pm 125 \AA^{2}$. The surface area per carboxylic acid group is probably much larger. With XPS, a 10-nm-thick layer is analysed and only part of the carboxylic acid groups will therefore be present at the surface.

\section{Immobilization of $\left[{ }^{14} \mathrm{C}\right]$ alb-hep conjugate onto (functionalized) PS surfaces - alb-hep surface concentration and stability}

The results of the immobilization of ${ }^{14} \mathrm{C}$-labelled albhep conjugate onto (functionalized) PS surfaces are shown in Figure 2. Alb-hep conjugate which is immobilized in the presence of EDC and NHS (E/N) on either a PS-Ar or a PS/C11(:)-Ar surface cannot be desorbed by SDS. Alb-hep conjugate which is immobilized in the absence of EDC and NHS is

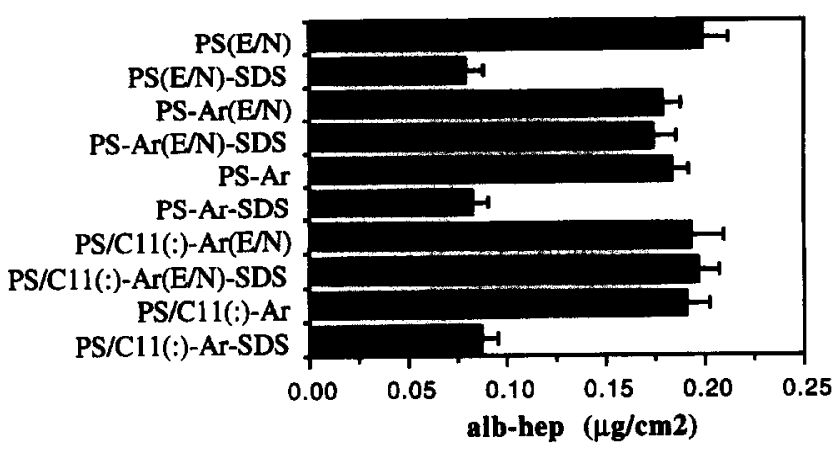

Figure 2 Surface concentration of $\left[{ }^{14} \mathrm{C}\right]$ alb-hep conjugate immobilized onto polystyrene [PS and PS/C11(:)] and argon plasma-treated polystyrene surfaces [PS-Ar and PS/C11(:)] in the absence or presence of EDC and NHS (E/N) before and after exposure to a $3 \%$ SDS solution. C11(:) represents the exposure of the surface to a solution containing the sodium salt of undecylenic acid $(0.3 \mathrm{M})$ prior to the plasma treatment; $n=3, \pm$ s.d.

Table 1 Elemental analysis of unmodified and argon plasma-treated (5s) polystyrene surfaces and argon plasma-treated surfaces reacted with trifluoroethanol

\begin{tabular}{|c|c|c|c|c|}
\hline Surface ${ }^{*}$ & O (at \%) & $\mathrm{Na}($ at $\%)$ & $F($ at \%) & $\% \mathrm{COOH}$ groups ${ }^{\dagger}$ \\
\hline \multirow[t]{2}{*}{ PS } & - & 0.34 & - & - \\
\hline & 0.05 & 0.20 & - & - \\
\hline PS-Ar & 11.9 & 0.26 & - & - \\
\hline \multirow{2}{*}{ PS-Ar- $\mathrm{H}_{2} \mathrm{O}-\mathrm{TFE}$} & 10.4 & - & 1.03 & 6.6 \\
\hline & 11.1 & - & 1.55 & 9.3 \\
\hline PS/C11(:) & $\begin{array}{l}6.49 \\
5.43\end{array}$ & $\begin{array}{l}4.12 \\
4.40\end{array}$ & - & E \\
\hline \multirow[t]{2}{*}{$\mathrm{PS} / \mathrm{CH}(:)-\mathrm{Ar}$} & 11.3 & - & - & - \\
\hline & 11.2 & - & - & - \\
\hline $\mathrm{PS} / \mathrm{C} 11(\mathrm{:})-\mathrm{Ar}-\mathrm{H}_{2} \mathrm{O}-\mathrm{TFE}$ & $\begin{array}{l}11.8 \\
10.5\end{array}$ & 二 & $\begin{array}{l}1.72 \\
1.72\end{array}$ & $\begin{array}{r}9.8 \\
10.9\end{array}$ \\
\hline
\end{tabular}

${ }^{*} \mathrm{C} 11(:)$ is a preadsorption of the sodium salt of undecylenic acid. $\mathrm{Ar}$, argon plasma-treated; $\mathrm{H}_{2} \mathrm{O}$, surfaces washed with water.

toxygen in $\mathrm{COOH}$ groups as percentage of the total amount of oxygen. 
physically adsorbed. Of the adsorbed conjugate, about $50 \%$ is desorbed after incubation in a $\mathbf{3} \%$ SDS solution. Approximately $60 \%$ of the alb-hep conjugate initially adsorbed onto the control surface (unmodified PS, not treated with an Ar plasma) is desorbed by the SDS desorption procedure.

\section{Immobilization of alb-hep conjugate on function- alized PS surfaces and the interaction of ATIII and thrombin with surface immobilized alb-hep conjugate studied with SPR}

The results of the immobilization of albumin and albhep conjugate, as well as the subsequent interactions of the surfaces with ATIII and thrombin as determined by SPR, are shown in Figure 3. Each step in the experiment is represented by a Roman number. Note that the $Y$-scale for these two experiments differs, which is due to differences in the settings of the SPR equipment. This has no consequences for the interpretation of the SPR results, since the difference in plasmon angle and not the plasmon angle itself is used in the calculation of the additional protein layer. The immobilization of albumin is somewhat faster than the immobilization of alb-hep conjugate (III), and the difference in SPR angle $\left(\theta_{\mathrm{pl}}\right)$ before and after the immobilization of albumin is larger than for the immobilization of alb-hep conjugate. The high ionic strength of the washing solutions $(>1 \mathrm{M})$ leads to large shifts of $\theta_{\mathrm{pl}}$ (IV). The applied washing procedure was such that the solution was taken out with a pipette, after which the cuvette was filled with another solution. The apparatus was left open during this procedure. This resulted in disturbances of the surface plasmon by light scattering. Therefore, the washing procedure is represented by a block-like type period. After adding ATIII to both compartments (V), a gradual shift of $\theta_{\mathrm{pl}}$ is observed for the alb-hep surface, whereas no $\theta_{\mathrm{pl}}$ shift for the albumin surface is observed. The last step, in which thrombin is added to both surfaces (VII), results in a large increase of $\theta_{\mathrm{pl}}$ for the alb-hep surface and a small increase for the albumin surface.

Figure 4 shows the results of an SPR experiment which was performed analogously to the one described above. After the addition of ATIII and the subsequent washing with PBS, an immunoglobulin against human ATIII was added, which results in a large plasmon angle shift for the alb-hep surface, whereas no shift is observed for the albumin surface.

\section{DISCUSSION}

The results obtained in this study show that alb-hep conjugate can be covalently immobilized onto argon plasma-modified PS surfaces. An argon plasma treatment of PS results in the introduction of carboxylic acid groups at the surface. The exact surface concentration of carboxylic acid groups cannot be calculated from the XPS measurements, because a layer of $\pm 10 \mathrm{~nm}$ is analysed. However, the amount of carboxylic acid groups present at the PS surface that can be activated by EDC and NHS and that can be used in the coupling of alb-hep conjugate is apparently high enough to covalently immobilize $\pm 0.2 \mu \mathrm{g} \mathrm{cm}^{-2}$ of $\left[{ }^{14} \mathrm{C}\right]$ alb-hep conjugate. This is about a theoretical (side-on) monolayer. During the immobilization procedure of alb-hep conjugate, a small amount of EDC and NHS is present. The stability of the alb-hep conjugate layer may therefore be attributed to both the covalent coupling of conjugate to the surface and to stabilization of the layer by cross-linking of the surface-bound conjugates. The contribution of crosslinking can be neglected because surfaces that were prepared by coupling of alb-hep conjugate to surfaces that were only preactivated with EDC and NHS were equally stable in a $3 \%$ SDS solution as surfaces to which alb-hep conjugate was coupled in the presence of EDC and NHS. Furthermore, the surface concentration of immobilized conjugate was the same for both procedures (results not shown). The surface concentration of immobilized conjugate $\left( \pm 0.2 \mu \mathrm{g} \mathrm{cm}^{-2}\right)$ is similar to the surface concentration of $\left[{ }^{14} \mathrm{C}\right]$ alb-hep immobilized onto carboxylated polystyrene beads ${ }^{16}$. Preadsorption of C11(:) results in a slightly higher

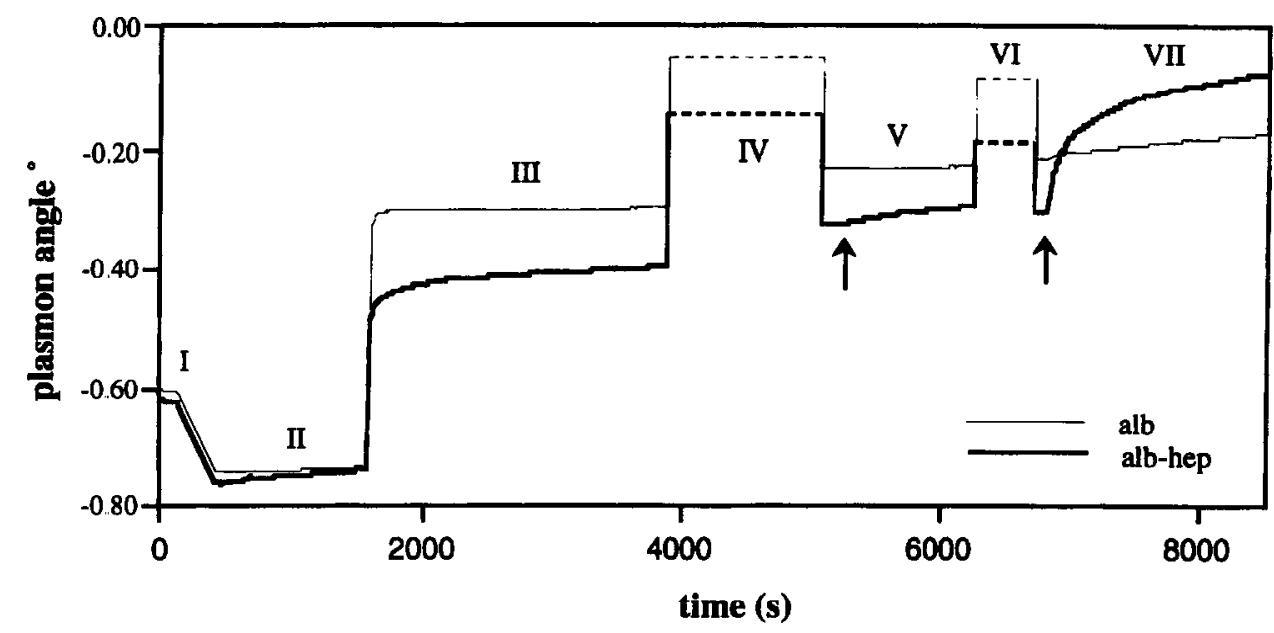

Figure 3 SPR experiment in which the interactions of ATIII and thrombin with surfaces containing either albumin or alb-hep conjugate immobilized on plasma-treated polystyrene surfaces are compared. Roman numbers represent different incubation steps. (I) PBS; (II) phosphate buffer containing EDC and NHS (1 mM); (III) immobilization of albumin or alb-hep conjugate; (IV) washing with solutions of high ionic strength; (V) replacement of the last washing solution by PBS, after which ATIII (arrow) was added (final conc. $100 \mu \mathrm{g} \mathrm{ml}^{-1}$ ); (VI) washing with PBS; (VII) addition of thrombin (arrow) to a PBS solution (final conc. $12.5 \mathrm{mU} \mathrm{ml}^{-}$). 


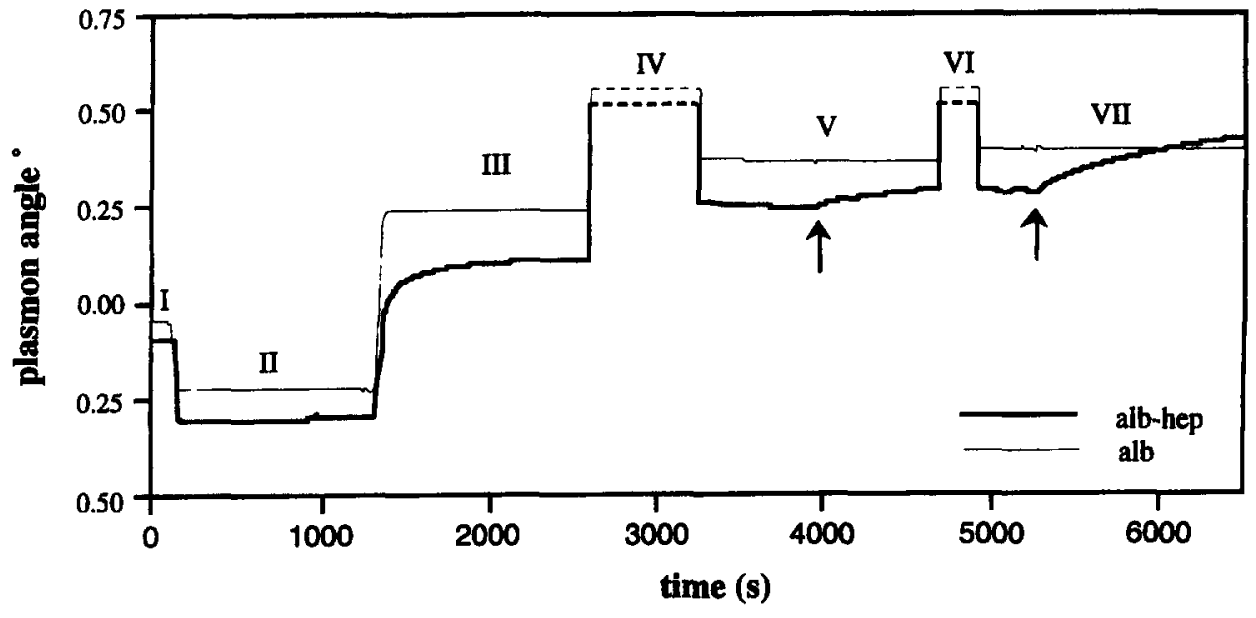

Figure 4 SPR experiment in which the SPR signal for binding of ATIII to an alb-hep surface is 'enhanced' by the binding of immunoglobulin G $\alpha$-ATIII to the surface-bound ATIII. Roman numbers represent different incubation steps. (I) PBS; (II) phosphate buffer containing EDC and NHS (1 mM); (III) immobilization of albumin or alb-hep; (IV) washing with solutions of high ionic strength; (V) replacement of the last washing solution by PBS, after which ATIII (arrow) was added (final conc. $100 \mu \mathrm{g} \mathrm{ml}^{-1}$ ); (VI) washing with PBS; (VII) addition of immunoglobulin G $\alpha-A T I I I$ (arrow) to a PBS solution ( $\pm 0.1 \mathrm{mg} \mathrm{ml}^{-1}$ ).

fluorine content on the PS surface after plasma treatment and the TFE reaction, as compared to a surface that was not exposed to the C11(:) solution. The higher fluorine content on the surfaces that were treated with C11(:) as compared to surfaces that were only treated with an argon plasma can be ascribed to the (covalent) immobilization of some of the adsorbed C11(:) molecules upon plasma treatment. In the present study, an argon plasma treatment alone was sufficient to immobilize a monolayer of alb-hep conjugate, as mentioned above. However, when smaller biomolecules or peptides are immobilized onto plasma functionalized surfaces, an additional amount of carboxylic acid groups at the surface may be beneficial to immobilize sufficient amounts of the biological compound. The immobilization of adsorbed C11(:) on PS surfaces with an argon plasma is currently optimized. It is possible that some of the alb-hep conjugate may be coupled via epoxides or aldehyde groups that are introduced by the plasma treatment, since not all of the conjugate immobilized without EDC and NHS was desorbed from plasma-treated PS surfaces. A more likely explanation for this is that the desorption procedure may not be sufficient to desorb all physically adsorbed conjugate.

The immobilization of alb-hep conjugate, as well as the interactions of ATIII and thrombin with the albhep surface, were monitored with SPR. In this study the interactions were studied under static conditions. The information about the rate of the interactions is therefore limited. For a good interpretation of the SPR results, the influence of the bulk refractive index on the plasmon angle should be considered. Replacing the buffered solution of EDC and NHS by an alb-hep conjugate or albumin solution of about $15 \mathrm{mg} \mathrm{ml}^{-1}$ results in an increase of the refractive index of the bulk solution and thus also of the layer near the surface that determines the plasmon angle. This change of $\theta_{\mathrm{pl}}$ depends on the protein concentration in the bulk and not on the immobilization of albumin or conjugate on the surface. Changing the buffer solution to a buffer solution with a lower ionic strength leads, similarly as after adding a concentrated protein solution, to a stepwise change of $\theta_{\mathrm{pl}}$. For instance, the decrease of the ionic strength in step I $\rightarrow \Pi$ (Figures 3 and 4 ) is accompanied by a decrease of $\theta_{\mathrm{pl}}$. Differentiation between the bulk effect and protein-surface interactions is possible when after each step the protein solution is replaced by a buffer solution, the plasmon angle of which has been determined before. The plasmon angle shift $\left(\Delta \theta_{\mathrm{pl}}\right)$ that corresponds with the immobilization of alb-hep conjugate can thus be derived from $\theta_{\mathrm{pl}}(\mathrm{V})-\theta_{\mathrm{pl}}(\mathrm{I})$.

The plasmon angle changes upon immobilization of albumin and alb-hep conjugate are very similar (Figures 3 and 4). However, with albumin a plateau level is reached almost instantaneously, whereas with alb-hep conjugate a plateau is reached more gradually. Since the SPR experiments were performed under static conditions, the rates of adsorption are diffusion limited. Albumin has a lower molecular weight than the conjugate and will thus diffuse somewhat faster to the surface. A factor that can decrease the immobilization rate of the alb-hep conjugate is the anionic character of the alb-hep conjugate. Alb-hep conjugate that encounters the surface, already covered with a certain amount of conjugate, may experience an electrostatic repulsion from the already adsorbed or immobilized alb-hep conjugate. Assuming a refractive index of 1.45, $\Delta \theta_{\mathrm{pl}}$ for immobilization of albumin and alb-hep can be converted into a layer thickness of 2.25 and $1.85 \mathrm{~nm}$ respectively ${ }^{7}$. The layer thickness of albhep conjugate $(1.85 \mathrm{~nm})$ should correspond with a surface coverage of $0.195 \mu \mathrm{g} \mathrm{cm}^{-2}$, as was determined with radiolabelled alb-hep conjugate. A surface coverage of $0.195 \mu \mathrm{g} \mathrm{cm}^{-2}$ corresponds to a side-on monolayer of conjugate. The dimensions of an albumin molecule are $4 \times 4 \times 14 \mathrm{~nm}^{14}$. A side-on monolayer of albumin will therefore result in a layer thickness of $\pm 4 \mathrm{~nm}$, whereas the thickness of a side-on monolayer of alb-hep conjugate will be around $4 \mathrm{~nm}$. The layer thickness derived from the SPR measurements assuming a refractive index of $\mathbf{1 . 4 5}$ is thus \pm two-fold less than that determined with the radioactivity measurements. It is possible that the refractive index of immobilized alb-hep conjugate and also of 
immobilized albumin differs from 1.45. The albumin and alb-hep conjugate are exposed to relatively high concentrations of EDC during preparation of the conjugate, which probably leads to internal crosslinking and conformational changes of the albumin molecule. These conformational changes, combined with possible conformational changes during immobilization, may result in a refractive index different from that of the native molecule. A small refractive index change gives a large change in the calculated layer thickness. The $\Delta \theta_{\mathrm{pl}}$ observed upon immobilization of alb-hep conjugate corresponds to a layer thickness of $4 \mathrm{~nm}$ when a refractive index of 1.39 is assumed for the alb-hep conjugates. Indeed, it was found that adsorbed albumin molecules have a lower refractive index than native molecules (unpublished data). The lower $\Delta \theta_{\mathrm{pl}}$ observed after alb-hep immobilization as compared to $\Delta \theta_{\mathrm{pl}}$ after albumin immobilization may be partially explained by the heparin content of the conjugate $(16 \% \mathrm{w} / \mathrm{w})$. It is known that glycoproteins and also lipoproteins have a somewhat lower refractive index than non-conjugated proteins, and therefore give a lower SPR response. Thus, for quantification of the SPR signals, one should determine both the refractive index of the adsorbed protein layer and the corresponding shift in the plasmon angle.

Since SPR is a non-destructive method, interactions of ATIII and thrombin with the same alb-hep and albumin surfaces could be investigated. The $\Delta \theta_{\mathrm{pl}}$ upon binding of ATIII to the alb-hep surface corresponds to a protein layer thickness of $\pm 0.25 \mathrm{~nm}$ assuming a refractive index of 1.45. It is expected that most of the surface-bound ATIII has retained its native conformation. This consideration is based on the fact that the majority of the ATIII molecules is bound to the heparin chain, which will not lead to denaturation of the ATIII molecule ${ }^{11}$. The dimensions of the ATIII molecule are $5.5 \times 5.5 \times 6.5 \mathrm{~nm}^{15}$. Thus, a layer thickness of $\pm 0.25 \mathrm{~nm}$ corresponds with $4-5 \%$ $\left( \pm 12 \mathrm{ng} \mathrm{cm}^{-2}\right)$ of a monolayer of ATIII $\left(0.25 \mu \mathrm{g} \mathrm{cm}^{-2}\right)$. The ATIII surface concentration, calculated with SPR, is somewhat higher than observed for the binding of ${ }^{14}$ C-labelled ATIII onto PS beads containing a monolayer of alb-hep conjugate ${ }^{18}$. The difference in ATIII binding in these two studies can be explained by differences in the conditions under which the binding of ATIII was performed. The ATIII binding experiment in the present study was performed at room temperature, whereas the binding experiments of $\left[{ }^{14} \mathrm{C}\right] \mathrm{ATIII}$ in the previous study were performed at $37^{\circ} \mathrm{C}$. Furthermore, in the previous study, the $\left[{ }^{14} \mathrm{C}\right]$ ATIII solution also contained albumin (27 $\mathrm{mg} \mathrm{ml}^{-1}$ ) and the washing procedures after the ATIII binding were more extensive. Nevertheless, the results of these two studies are qualitatively the same. Both studies show that only a small fraction of the heparin molecules at the surface is able to bind ATIII from solution. The absence of ATIII at the albumin surface confirms that the interaction between ATIII and the alb-hep surface is mediated by the immobilized heparin.

Because the $\Delta \theta_{\mathrm{pl}}$ of ATIII depends on the mean refractive index change near the sensor surface, small proteins will give a smaller signal than larger proteins. Binding of immunoglobulin G $\alpha$-ATIII to ATIII molecules at the alb-hep surface (VII) results in an
SPR signal which is \pm three-fold larger than the signal for the binding of ATIII (V). This ratio corresponds roughly with the ratio of the molecular weights of these two proteins ( 58 versus $150 \mathrm{kD}$ ). Immunoglobulins may also be useful when protein mixtures are exposed to a surface of interest. Since the plasmon angle shift is built up by the interactions of several proteins with the surface, one cannot determine the contribution of separate proteins. Using polyclonal antibodies against the proteins at the surface, the relative surface concentrations of previously adsorbed proteins may be determined. Monoclonal antibodies against specific epitopes can be used to determine the conformation of surface-bound proteins. To confirm, for instance, that ATIII molecules are bound to the immobilized heparin on the alb-hep surface, one should make use of a monoclonal antibody against the site in ATIII that is involved in the binding to heparin.

When thrombin is exposed to the alb-hep surface that contains some ATIII, a large shift in $\theta_{\mathrm{pl}}$ is observed, which corresponds with an additional layer thickness of $1.5 \mathrm{~nm}^{3}$. The adsorption of thrombin on the albuminated surface is much lower and probably nonspecific, as it shows a low-affinity character. In this particular experiment, thrombin may bind to surfacebound ATIII forming a thrombin-ATIII complex, or it can bind to 'free' heparin chains on the surface not complexed with ATIII. From the large shift in $\Delta \theta_{\mathrm{pl}}$, which is also observed when the alb-hep is not preadsorbed with ATIII (data not shown), it can be concluded that the immobilized alb-hep conjugate is able to bind much more thrombin than ATIII. A similar observation was made in an earlier study in which the interactions between ATIII, thrombin and surface-immobilized heparin were investigated with ${ }^{14}$ C-labelled ATIII and a chromogenic substrate for thrombin ${ }^{16}$. The higher thrombin surface concentration as compared to the surface concentration of ATIII can be explained by the less specific heparin-thrombin interaction as compared to the heparin-ATIII interaction.

\section{CONCLUSIONS}

In the present study, the immobilization of alb-hep conjugate on a functionalized PS surface and the interactions of this surface with ATIII and thrombin were monitored with SPR. ATIII could be bound to the alb-hep surface but not to an albuminated PS surface. Binding of an immunoglobulin against human ATIII to the alb-hep surface previously exposed to ATIII confirmed the presence of ATIII on the heparinized surface. The alb-hep immobilized surface was able to bind much more thrombin than ATII, which is probably due to the less specific heparin-thrombin interaction as compared to the heparin-ATIII interaction.

The results obtained with SPR with regard to the immobilization of alb-hep conjugate differ quantitatively from the results obtained with radiolabelled conjugate but are qualitatively the same. The quantitative difference between these two methods probably arose from the uncertainty of the refractive index of the adsorbed proteins layer. However, this study shows that plasma treatment of polymer surfaces and 
SPR is a powerful combination that can be used to study, for instance in real time, the heparinization of polymer surfaces and the subsequent interactions of the modified surfaces with proteins.

\section{ACKNOWLEDGEMENTS}

D.J. van de Heuvel and A.T.M. Lenferink are acknowledged for their help with the SPR experiments. R.A. van Boxtel is acknowledged for performing initial SPR experiments.

\section{REFERENCES}

1. Liedberg, B., Nylander, C. and Lundström, I., Surface plasmon resonance for gas detection and biosensing. Sensors and Actuators, 1983, 4, 299-304.

2. Jönsson, U. and Malmqvist, M., Real time biospecific interaction analysis, the integration of surface plasmon resonance detection, general biospecific interface chemistry and microfluids into one analytical system. Adv. Biosensors, 1992, 2, 291-336.

3. Reather, H., Surface plasmons. Springer Tracts in Modern Physics, Vol. 11. Springer, Berlin, 1988.

4. Stenberg, E., Persson, B., Roos, H. and Urbaniczky, C. Quantitative determination of surface concentration of proteins with surface plasmon resonance using radiolabeled proteins. J. Coll. Interface Sci., 1991, 143(2), 513-526.

5. Kooyman, R. P.H., Lenferink, A. T.M., Eenink, R. G. and Greve, J., Vibrating mirror surface plasmon resonance immunosensor. Analyt. Chem., 1991, 63, 83-85.

6. Lenferink, A.T.M., Kooyman, R.P.H. and Greve, J., An improved optical method for surface plasmon resonance experiments. Sensors and Actuators B, 1991, 3, 261-265.

7. Kooyman, R.P. H., Kolkman, H., Gent, J. van and Greve, J., Surface plasmon resonance immunosensors: sensitiv- ity considerations. Anal. Chim. Acta, 1988, 213, 35-45.

8. Mayo, C. S. and Hallock, R. B., Immunosssay based on surface plasmon oscillations. J. Immunol. Meth., 1989, 120, 105-114.

9. Terlingen, T.C.A., Brenneisen, L.M., Super, H.T. J., Pijpers, A. P., Hofman, A.S. and Feijen, J., Introduction of amine groups on poly(ethylene) by plasma immobilization of a preadsorbed layer of decylamine hydrochloride. J. Biomater. Sci., Polym. Edn, 1994, 4(3), 165-181.

10. Chilkoti, A., Ratner, B.D. and Briggs, D., Plasmadeposited polymeric films from carbonyl-containing volatile precursor: XPS chemical derivatization and static SIMS surface characterization. Chem. Mater. $1991,3,51-61$.

11. Terlingen, J. G. A., Hofman, A.S. and Feijen, J., Effect of glow discharge treatment of poly(acrylic acid) preadsorbed onto polyethylene. I. Appl. Polym. Sci, 1993, 50, 1529-1539.

12. Brandrup, J. and Immergut, E.H. (eds), Polymer Handbook, 3rd edn. John Wiley, New York, 1989.

13. Engbers, G.H.M., The development of heparinized surfaces with an improved blood compatibility, Chapter 3. Thesis, University of Twente, 1990.

14. Creighton, T. E., In Proteins. W. H. Freeman, New York, 1983.

15. Mourey, L., Samama, J.-P., Delarue, M., Petitou, M., Choay, J. and Moras, D., Crystal structure of cleaved bovine antithrombin III at $3.2 \AA$ resolution. J. Molec. Biol., 1993, 232, 223-241.

16. Delden, C.J. van, Engebers, G.H.M. and Feijen, J., Interactions between antithrombin III, thrombin, and surface immobilized heparin. J. Biomater. Sci., Polym. Edn, 1996, 7, 989-1004.

17. Multihaup, G., Bush, A. I., Pollwein, P., Masters, C. L. and Beyreuther, K., Specific binding of the Alzheimer $\beta$ A4 amyloid precursor to collagen, laminin and heparin. Proceedings 2nd European BLA Symposium, 1992, pp. 17-19.

18. Delden, C. J. van, Engbers, G.H.M. and Feijen, J. Interaction of antithrombin III with surface immobilized albumin-heparin conjugates. J. Biomed. Mater. Res., 1995, 29, 1317-1329. 